\title{
Shape changing and accelerating solitons in integrable variable mass sine-Gordon model
}

\author{
Anjan Kundu \\ Theory Group, Saha Institute of Nuclear Physics \\ Calcutta, INDIA \\ anjan.kundu@saha.ac.in
}

September 11, 2021

\begin{abstract}
Sine-Gordon model with variable mass (VMSG) appears in many physical systems, ranging from the current through nonuniform Josephson junction to DNA-promoter dynamics. Such models are usually nonintegrable with solutions found numerically or peturbatively. We construct a class of VMSG models, integrable both at classical and quantum level with exact soliton solutions, which can accelerate, change their shape, width and amplitude simulating realistic inhomogeneous systems at certain limits.
\end{abstract}

PACS: 05.45.Yv,03.65.Fd,11.10.Lm,11.55.Ds

Sine-Gordon (SG) model enjoys a special status among nonlinear integrable systems for its inherent richness and wide range of applications in different fields $[1,2,3,4,5,6,7,8,9]$. Apart from the fascinating properties of an integrable system, the SG model exhibits relativistic invariance and integervalued topological charge represented by solutions like kink, antikink, breather etc. [10], together with the quantum integrability described by the Yang-Baxter equation (YBE), which for the SG leads to quantum algebra $s u_{q}(2)[11,12]$.

Solitons in the standard SG model, as in other integrable systems, move with constant velocity and shape. In realistic situations however due to inhomogeneity of the media solitons may exhibit more complex motion with changing velocity and shape $[4,6,7]$, which may be used also as a desirable effect for fast transport, fast communication, or even for a possible soliton gun [8]. In particular inhomogeneity can lead to SG models with variable mass (VMSG) in describing fluxon or semi-fluxon dynamics in Josephson junction (JJ) with impurity or nonuniform critical current $[2,6]$, spin wave propagation with variable interaction strength [5], DNA-promoter dynamics in nonuniform background etc. However such inhomogeneities usually destroy the most cherishable property of the SG model, i.e. its integrability and hence the solutions can be extracted only numerically or at best peturbatively $[2,4,6,7]$.

We observe that, though the integrability of SG model is spoiled by a variable mass, or a variable velocity, it can be restored if both of them vary simultaneously following certain rule. Therefore we can construct a VMSG model, integrable at the classical and the quantum level, allowing analytic soliton solutions. Such exact solitons nevertheless show intriguing accelerated motion with changing shape, amplitude and width, simulating thus realistic inhomogeneous systems $[4,6,7]$ and describing them analytically at certain limits (Fig. 1b-d).

To clarify our strategy we focus on the linear spectral problem of the SG model: $\Phi_{x}(x, \lambda)=$ $U(\lambda, x) \Phi(x, \lambda)$

$\Phi_{t}(x, \lambda)=V(\lambda, x) \Phi(x, \lambda)$, with its Lax pair [13]: $\quad U=\frac{i}{4}\left(-u_{t} \sigma^{3}+m k_{1} \cos \frac{u}{2} \sigma^{2}-m k_{0} \sin \frac{u}{2} \sigma^{1}\right), \quad$ and 
$V=\frac{i}{4}\left(-u_{x} \sigma^{3}-m k_{0} \cos \frac{u}{2} \sigma^{2}+m k_{1} \sin \frac{u}{2} \sigma^{1}\right)$, where $k_{0}(\lambda)=2 \lambda+\frac{1}{2 \lambda}, \quad k_{1}(\lambda)=2 \lambda-\frac{1}{2 \lambda}$, with spectral parameter $\lambda$. Compatibility condition $\Phi_{x t}=\Phi_{t x}$ or the related flatness condition leads to the SG equation, for constant mass $m_{0}$ and spectral parameter $\lambda_{0}$. Recall that in the inverse scattering (IS) method solitons are obtained at discrete spectrum $\lambda_{n}, n=1,2, \ldots N$, with velocities of SG solitons linked to these values of the spectral parameter. Therefore variable soliton velocity should have a variable spectral parameter $\lambda$, which alone however violates the compatibility condition. Fortunately by making mass $m$ also a space-time dependent variable, we can get the VMSG equation

$$
u_{t t}-u_{x x}+m^{2}(x, t) \sin u=0,
$$

with the constraint: $\left(k_{0} m\right)_{t}+\left(m k_{1}\right)_{x}=0,\left(k_{1} m\right)_{t}+\left(m k_{0}\right)_{x}=0$, which can be reduced to two free field equations:

$$
\kappa_{t t}-\kappa_{x x}=0, \rho_{t t}-\rho_{x x}=0, \text { for } \kappa=\ln m(x . t), \rho=\ln \lambda(x, t) .
$$

Note that the set of equations (1-2) is a new integrable relativistic system, generalizing SG equation, and a reduction (at the free field limit of spectral dilatation field $\rho$ ) of the conformal affine Toda model (CATM) [14]. However since we are interested here in application to physically relevant inhomogeneous models, we would consider $\kappa, \rho$ as given inhomogeneous functions by restricting to particular solutions for variable mass and spectral parameter:

$$
m(x, t)=m_{0} f_{+} f_{-}, \quad \lambda(x, t)=\lambda_{0} \frac{f_{+}}{f_{-}}
$$

with $f_{ \pm}$arbitrary smooth functions of $x_{ \pm}=x \pm t$, respectively. Thus we obtain an integrable SG equation (1) with variable mass $m(x, t)=m_{0} f_{+} f_{-}$. Note that due to explicit space-time dependent coefficient, it is no longer a relativistic and translational invariant model. However if we demand such invariance, we simply get back the constant mass SG model, as shown in [15]. We can control mass $m(x, t)$ in the VMSG model (1) by choosing suitably the inhomogeneity functions $f_{ \pm}$for different physical situations, showing a variety of soliton dynamics as in Fig. 1 a-d.

For obtaining exact solutions of the VMSG model (1), we can apply well known methods designed for integrable systems [10], e.g. Hirota's bilinearization and the IS formalism. Hirota's method for soliton solution for the standard SG equation is given through a cleverly chosen ansatz $u=-2 i \ln \frac{g_{+}}{g_{-}}$, with $g_{ \pm}$as conjugate functions, which converts the SG equation into a bilinear form, admiting solution for $g_{ \pm}$as expansion in plane-waves. For SG model (1) with variable mass and velocity the same ansatz seems to work, only the plane waves should be replaced by their generalized form: $g^{(n)}=\frac{c_{n}}{\lambda_{n}} e^{\frac{i}{2}\left(X\left(\lambda_{n}, x, t\right)-T\left(\lambda_{n}, x, t\right)\right)}$, where $X\left(\lambda_{n}, x, t\right)=\int^{x} d x^{\prime} m\left(x^{\prime}, t\right) k_{1}\left(\lambda_{n}, x^{\prime}, t\right), T\left(\lambda_{n}, x, t\right)=$ $\int^{t} d t^{\prime} m\left(x, t^{\prime}\right) k_{0}\left(\lambda_{n}, x, t^{\prime}\right)$. This gives the exact soliton solutions of (1) through the expansion: $g_{ \pm}=$ $1 \pm g^{(1)}, \quad$ for 1-kink and $g_{ \pm}=1 \pm\left(g^{(1)}+g^{(2)}\right)+s\left(\frac{1}{2}\left(\theta_{1}-\theta_{2}\right)\right) g^{(1)} g^{(2)}, \lambda_{a}=\frac{i}{2} e^{\theta_{a}}, a=1,2$ for 2-kink etc., with the scattering amplitude $s(\theta)=\tanh ^{2} \theta$, while for $\lambda_{2}=-\lambda_{1}^{*}=\eta e^{i \theta}$, one gets the kink-antikink bound state (breather solution).

Similarly we can apply the IS formalism [10] to (1), for which the crucial step is to use the analyticity of Jost solutions $\Phi$, based on their behavior at $\lambda \rightarrow \pm \infty$. This holds equally for the inhomogeneous extension of the SG model, where the asymptotic plane waves should again be replaced by their generalized form. 1-kink soliton with $\lambda_{1}=i \eta$ can be obtained explicitly, either from the Hirota's or from the IS method as

$$
u=4 \tan ^{-1}\left(e^{\zeta}\right), \zeta=\frac{i}{2}(X(i \eta, x, t)-T(i \eta, x, t)),
$$

with variable soliton velocity $v_{s}(x, t)=-\frac{d x}{d t}=\frac{k_{1}(\eta, x . t)}{k_{0}(\eta, x . t)}$. Kink solution (4) gives a localized soliton for $\sin \frac{u}{2}=\frac{1}{\cosh \zeta}$, which are actually shown in Fig 1 . 
To see the effect of various inhomogeneities on the soliton dynamics, we consider some concrete integrable cases. Notice that variable mass i) $m_{0}\left(x^{2}-t^{2}\right)^{n}$, invariant under relativistic motion, yields ( for $n=1$ ) the exact kink solution (4) with $\zeta=\frac{m}{3}\left(2 \eta(x-t)^{3}+\frac{1}{2 \eta}(x+t)^{3}\right)$, the evolution of the corresponding soliton is depicted in Fig 1a. The intriguing change in the soliton shape, width and velocity during its motion is clearly seen. Position-dependent mass can be achieved in this case at $t \rightarrow 0$ and therefore phenomenon like fluxon propagation through JJ with local defect $m_{0} x^{2}$ may be described with the above analytic soliton solution at a short time interval limit, as shown in Fig $1 \mathrm{~b}$.

Other forms of integrable VMSG equations can be obtained for mass ii) $\sqrt{2} m_{0} \cos ^{\alpha} q(x \pm t), \alpha$ being an arbitrary parameter. We derive for the first case (with $\alpha=1$ ) kink solution (4) with $\zeta=m_{0}\left(k_{0}(\eta) x-k_{1}(\eta) t+\frac{1}{4 q \eta} \sin 2 q(x+t)\right)$, having soliton velocity $v_{s}=m_{0} d\left(k_{1}(\eta)-\frac{1}{2 \eta} \cos 2 q(x+t)\right)$ and width $d=\left(m_{0}\left(k_{0}(\eta)+\frac{1}{2 \eta} \cos 2 q(x+t)\right)\right)^{-1}$, both of which oscillate periodically in space-time, as evident from Fig. 1c. Notice that variable mass of this type is particularly interesting, since it can describe an important physical situation, namely the SG equation parametrically driven by a plane wave [17].

One can get a similar integrable case with mass iii) $m_{0}(2 \cos q(x+t) \cos q(x-t))^{\frac{\alpha}{2}}$, which at short time interval limit $(t \rightarrow 0)$ gives $\approx m(x)=\tilde{m}_{0}(\cos q x)^{\alpha}$, while for evolution limited to a small space interval $(x \rightarrow 0): \approx m(t)=\tilde{m}_{0}(\cos q t)^{\alpha}$. Recall that a physically motivated spin chain with coupling constant changing periodically in space, can be described by a VMSG with mass $m(x)=m_{0}(\cos q x)^{\alpha}$, where $\alpha=\frac{1}{2-K}$, with $K \geq \frac{1}{2}$ being an important parameter of the system [5]. Similarly a real oscillator chain pumped by an alternating current [16] can be linked to a VMSG with mass $m(t)=$ $(\cos q t)^{\frac{1}{2}}$. Therefore we may conclude that the analytic solution of our VMSG equation can describe the inhomogeneous spin wave dynamics [5] or evolution of forced oscillators [16], at least at short time or space interval limit. Alternatively, this realistic spin (or oscillator) model can be tuned to the integrable VMSG with mass $m_{0} \cos ^{\alpha} q(x+t)$, by making its coupling strength oscillate periodically also in time (or space).

In most physical situations the inhomogeneity of the media leads to VMSG equation, with only position-dependent mass $m(x)$. Therefore we explore to find, when such equations can be integrable in the entire space-time and conclude from our result (3), that the VMSG becomes integrable only for the space-dependent mass iv) $m(x)=m_{0} e^{\rho\left(x-x_{0}\right)}$ with $\rho=$ const., which explains also why most of the realistic VMSG models having a different position-dependent mass (e.g. [5]) turn out to be nonintegrable systems. Exact kink solution for the integrable $m(x)$ is obtained from (4) as $u=$ $4 \tan ^{-1}\left(e^{\zeta}\right), \zeta=\frac{1}{\rho} k_{0}(t) m(x), m(x)=\exp \left(\rho\left(x-x_{0}\right)\right), k_{0}(t)=\cosh \left(\theta-\rho\left(t-t_{0}\right)\right)$. The corresponding soliton velocity and width are $v_{s}=\tanh \left(\theta-\rho\left(t-t_{0}\right)\right)$, and $d=\left(m(x) k_{0}(t)\right)^{-1}$, showing how the soliton shape changes and how it accelerates, decelerates or can exhibit boomeron [18] like property. This scenario is close to the predicted behavior of solitons in the dynamically active promoter zone of the $\mathrm{T}_{1}$ DNA [4]. Notice that for $\rho>0$, since $m(\infty)=\infty, m(-\infty)=0$, the kink solution yields $u(\infty)=$ $2 \pi, u(-\infty)=\pi$, and hence corresponds to the topological charge $Q=\frac{1}{2 \pi}(u(\infty)-u(-\infty))=\frac{1}{2}$. This intriguing fact might serve as an integrable theory for the semi-fluxon, observed in unconventional JJ [19].

At $\rho \rightarrow 0: \zeta \rightarrow \zeta_{0}=m_{0}\left(k_{0}\left(x-x_{0}\right)-k_{1}\left(t-t_{0}\right)\right)$ and the standard SG soliton is recovered. Therefore we can acess the solitonic behavior in realistic models with any mass deviation from its constant value, with high degree of accuracy, by approximating through expansion in powers of $\rho$. Fig. 1d shows that a static soliton remains static, when placed in a region with constant mass while an initially static soliton can move with accelerated (or decelerated) motion, when placed in a zone with variable mass, resembling closely the scenario of the VMSG soliton in the DNA chain, which with zero initial velocity in an inactive regions (with constant mass due to almost uniform background of two types of base pairs) remains static, while in the active promoter region with variable mass (due to significant difference in the number of lighter (A-T) and heavier (G-C) base pairs) the same static 

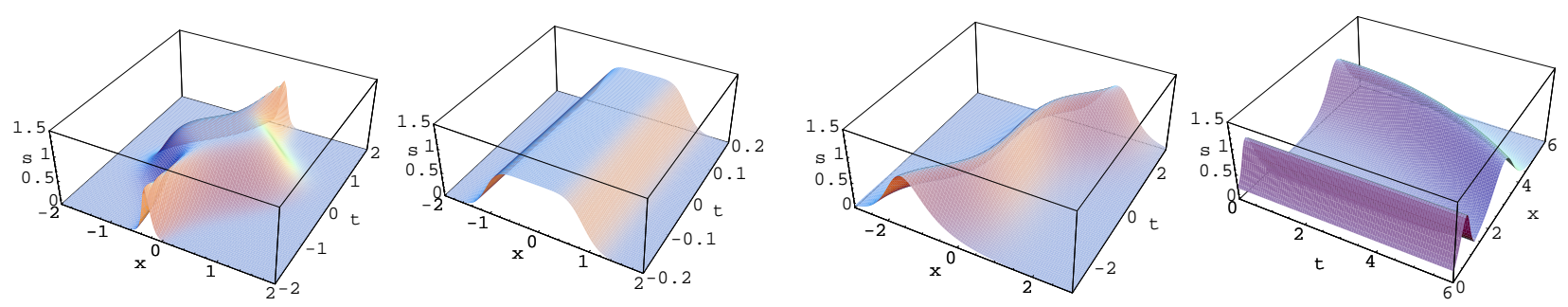

Figure 1: Exact soliton solutions: $s=\sin \frac{u}{2}(x, t)$ of the integrable VMSG equation with variable mass m. a) $m=m_{0}\left(x^{2}-t^{2}\right)$ having an intriguing flattening of the soliton at the center. b) Short time interval limit of the above soliton showing the flattening prominently. c) $m=2 m_{0} \cos q(x+t)$ with oscillatory behavior of the soliton. d) Static soliton in the zone $(x \leq 1.2)$ with $m=$ const and initially static soliton in the zone (at $x=4.8$ ) with variable mass: $m=m_{0} \exp (\rho x) \approx m(1+\rho x)$, with $\rho=0.1$ moves backward with acceleration, resembling soliton propagation in inactive/active promoter region in a DNA chain.

soliton can acquire rich accelerated motion [4].

Finally we intend to show that the integrable VMSG model constructed here can be raised to the quantum level and the algebraic Bethe ansatz (ABA) developed for the constant mass SG model [11] can be adopted successfully for it. Quantum lattice regularized SG matrix Lax operator $U_{j}\left(\lambda, \mathbf{S}_{j}, m\right), j=1,2, \ldots, L$ involves quantum-spin operators $S_{j}^{3}\left(u_{j}\right), S_{j}^{ \pm}\left(u_{j}, p_{j}, m\right)$, expressed in canonical operators $u_{j}, p_{j}=\dot{u}_{j}$ and the mass parameter $m$, which should be generalized now to site dependent parameter $m_{j}[20]$. Note that, the trigonometric $R\left(\frac{\lambda}{\mu}\right)$-matrix associated with our quantum integrable VMSG model remains the same, since it depends on the ratio of two spectral parameters, in which $x, t$-dependence enters, as seen from (3), only multiplicatively and therefore gets canceled. Moreover, YBE being a local algebra (at each lattice site $j$ ) is not affected by inhomogeneity and yields the same quantum algebra $s u_{q}(2)$; only with the replacement of constant $m$ by a site-dependent function $m_{j}$ in its structure constant: $\left[S_{j}^{+}, S_{k}^{-}\right]=\delta_{j k} m_{j} \frac{\sin \alpha 2 S_{j}^{3}}{\sin \alpha}$.

The aim of ABA is to solve exactly the eigenvalue problem of $\operatorname{tr} T(\lambda)=A(\lambda)+D(\lambda)$, with $T(\lambda)=\prod_{j} U_{j}(\lambda)$, generating all conserved operators including the Hamiltonian, with the eigenstates: $\left|\lambda_{1}, \ldots, \lambda_{n}>=\prod_{j}^{n} B\left(\lambda_{j}\right)\right| 0>. T_{12}(\lambda)=B(\lambda)$ acts as creation operator, while $T_{21}(\lambda)=C(\lambda)$ as destruction operator annihilating the pseudovaccum: $C(\lambda) \mid 0>=0$. Following closely [11], but generalizing for the site-dependent mass $m_{j}$, we can construct the local pseudovaccum $\left|0>=\prod_{j}\right| \Omega_{j}^{(2)}>$, a crucial step in ABA, by combining the action of consecutive pair of Lax operators: $U_{j} U_{j+1} \mid 0>[21]$. Consequently the vacuum eigenvalues are generalized for the quantum VMSG model as $A(\lambda) \mid 0>=$ $\alpha_{(m)}|0>, \quad D(\lambda)| 0>=\beta_{(m)} \mid 0>$, where $\alpha_{(m)}=\prod_{j} a\left(\theta, \frac{m_{j}}{m_{j+1}}\right), \beta_{(m)}=\prod_{j} a^{*}\left(\theta, \frac{m_{j+1}}{m_{j}}\right)$ with $a\left(\theta, \frac{m_{1}}{m_{2}}\right)=$ $\frac{m_{1}}{m_{2}}+\delta^{2} m_{1} m_{2}(\cosh (2 \theta+i \alpha))$. This yields the exact eigenvalue for the conserved quantities: $\operatorname{tr} T(\lambda)$ as $\Lambda\left(\lambda ; \lambda_{1}, \ldots, \lambda n\right)=\alpha_{(m)} \prod_{j}^{n} f\left(\frac{\lambda_{j}}{\lambda}\right)+\beta_{(m)} \prod_{j}^{n} f\left(\frac{\lambda}{\lambda_{j}}\right)$, where $f\left(\frac{\lambda}{\mu}\right)$ is expressed through the elements of the $R\left(\frac{\lambda}{\mu}\right)$-matrix, which remains unchanged for the VMSG model. The Bethe equations for determining the parameters $\left\{\lambda_{j}\right\}$ are generalized similarly by taking $m \rightarrow m_{j}$.

Since our VMSG model can be reduced from CATM, a coordinate transformation $(x, t) \rightarrow(X, T)$ exists, which can take the SG model with variable mass formally to the SG model with constant mass, though the domain might shift to an unphysical region and singularities might arise. Such a nonlinear transformation, amounting to going to a noninertial frame, takes particularly simple form in the light-cone coordinates : $X_{ \pm}=\int d x_{ \pm} f_{ \pm}^{2}[15,14]$. However for investigating the physical effect of a given inhomogeneous medium inducing accelerated and shape changing solitons, one has to analyze the model in its original form with variable mass. Similar situation arises also in other inhomogeneous 
systems with integrable nonisospectral flow, e.g. [22] in the study of accelerated solitons in plasma through NLS equation, in discrete NLS, in Toda chain, in matrix Schrödinger problem with boomeron solution [18] etc. In most of these models though the inhomogeneities could be removed by tricky nonlinear transformations, the investigations were carried out in the original systems due to their physical relevance. Surprisingly this long list of literature devoted to various inhomogeneous integrable models does not include the well known SG model and also avoids any quantum treatment, except perhaps a recent attempt [23]. This enhances therefore the importance of the present result, which explores the inhomogeneous integrable SG model, presents its exact solution both in the classical and in the quantum case. Its analytic soliton solutions can simulate at certain limits realistic events like, fluxons in nonuniform Josephson junction, dynamics of spin waves with variable coupling, DNA solitons in the active promoter region etc. Regulating the position-dependent mass in integrable VMSG model one can create semi-kink solution suggesting a possible exact theory for semi-fluxon.

The author likes to thank Profs. P. Mitra (SINP) and D. Sen (IISC) for helpful discussions.

\section{References}

[1] M. Remoissenet, Waves called Solitons (Springer, Berlin, 2003); A. Ludu,Nonlinear waves and solitons on contours and closed surfaces (Springer, Berlin, 2007); V. G. Baryakhtar, Dynamics of topological magnetic solitons, (Springer, Berlin, 1994); A Barone, G. Palerno, Physics and applications of Josephson effect, (Wiley, NY, 1982); M. J. Rice et al, Phys. Rev. Lett. , 36, 432 (1976); G.L. Lamb, Rev. Mod. Phys. 43, 99 (1971)

[2] E. Goldobin et al , Phys. Rev. Lett. , 92, 057005 (2004); H. Susanto and S. A. van Gils, Phys. Rev. , 69, 092507 (2004);

[3] L. V. Yakushevich, Nonlinear Physics of DNA, (Chicheler, 2004); S. N. Englander et al, Proc. Nat. Acad. Sc. 77, 7222 (1980)

[4] M. Salerno, Phys. Rev. A 44, 5292 (1991);

[5] D. Sen and S. Lal, Eur. Phys. Lett. 52, 337 (2000)

[6] O. M. Braun, Y. S. Kivshar, The Frenkel-Kontorova model (Spinger, 2004); L. Munteanu, S. Dnescu, Introduction to soliton theory: Application in mechanics (Springer Maths, 2004); T. Dauxois, Physics of solitons (Cambridge Univ. Press., 2006) ; Soliton-driven photonics (Ed. A. D. Boardman et al, Springer, 2001); A. R. Bishop, Solitons in Action , (Acad. Press, NY, 1978), ch. 4, p 72; R. D. Parmentier, ibid, ch. 8, p 186; D. W. McLaughlin and A. C. Scott, , ibid, ch. 9, p 186

[7] M. Cadoni et al, arXiv:hep-th/0702213 (2007); N. Theodorakopoulos and E. W. Weller, Phys. Rev. B 38, 2749 (1988); N. F. Pedersen et al, Phys. Rev. B 30, 4057 (1984); J. C. Fernandez et al, Phys. Rev. Lett. 46, 753 (1981);

[8] N. Riazi and A. Sheykhi, Phys. Rev. D 74, 025003 (2006)

[9] M. Cholascinski and R. W. Chhajlany, Phys. Rev. Lett., 98, 127001 (2007)

[10] L. D. Faddeev and L. A. Takhtajan, Hamiltonian method in the theory of solitons (Springer, 2007); S. Novikov et al Theory of solitons (Plenum, N.Y., 1984) ; M. Ablowitz and H. Segur, Solitons and inverse scattering transform (SIAM, Philadelphia, 1981) ; R. Hirota, The direct method in soliton theory (Cambridge Univ. Press., 2004) 
[11] E. Sklyanin et al, Teor.Mat.Fiz. 40, 194 (1979)

[12] L. D. Faddeev, Int. J. Mod. Phys. 10, 1845 (1995)

[13] M. Ablowitz et al, Phys. Rev. Lett., 30, 1262 (1973); V.E. Zakharov et al Dokl. Akad. Nauk SSSR 219, 1334 (1974); D. Kaup, Stud. Appl. Math. 54, 165 (1975)

[14] O. Babelon and L. Bonora, Phys. Lett B 244, 220 (1990); H. Aratyn et al, Phys. Lett. B 254, $372(1991)$

[15] I.V. Barashenkov and B. S. Getmanov, Comm. Math. Phys. 112, 423 (1987)

[16] Y. Braiman, Nature 378, 465 (1995); A. Gavrielides et al, Phys. Rev. E 58 , 5529 (1998)

[17] I.V. Barashenkov et al, Europhys. Lett. 15, 113 (1991)

[18] F. Calogero and A. Degasperis, in Solitons (Ed. R. Bullough and P. Caudrey, Springer, Berlin, 1980), p 323

[19] H. Hilgenkamp et al, Nature 422, 50 (2003); T. Gaber et al, Phys. Rev. B 72, 054522 (2005)

[20] A. Kundu, Phys. Rev. Lett. 82, 3936 (1999)

$[21] \mid \Omega_{j}^{(2)}>=\quad\left(1+\delta^{2} g_{m_{2 j} m_{2 j+1}}\left(q_{2 j}, q_{2 j+1}\right)\right) f_{m_{2 j} m_{2 j+1}}\left(q_{2 j}, q_{2 j+1}\right), \quad$ where $\quad f_{m_{1} m_{2}}=$ $\left(m_{2}^{\frac{q_{2}}{\alpha}} / m_{1}^{\frac{q_{1}}{\alpha}}\right) f_{m}, g_{m_{1} m_{2}}=\left(\frac{m_{2}}{m}\right)^{2} g_{m}$, are generalizations over their known solutions $f_{m}, g_{m}$ with mass $m=$ const. [11]

[22] H. H. Chen and C. S. Liu, Phys. Rev. Lett. 37, 693 (1976); R. Balakrishnan, Phys. Rev. A 32, 1144 (1985); V. N. Serkin et al, Phys. Rev. Lett. 98, 074102 (2007) R. Scharf and A. R. Bishop, Phys. Rev. A 43, 6535 (1991); V.V. Konotop et al, Phys. Rev. E 48, 563 (1993) ; O. Ragnisco and R. Levi, Univ. Roma preprint No. 762 (1990)

[23] A. Kundu, Phys. Lett. B 633, 657 (2006) 